\title{
Top-down fabrication of silicon nanowire sensor using electron beam and optical mixed lithography
}

\begin{abstract}
The realization of reliable nanobiosensor devices requires the improvement of fabrication techniques to form the nanometer-sized structures and patterns, which were used to attach nano materials such as DNA for the device elements. This study demonstrates the sensitivity of silicon nanowires (SiNWs) as a sensing element in sensor application. Starting with silicon on insulator (SOI) material, the SiNWs with $<100 \mathrm{~nm}$ in width were fabricated using electron beam lithography combined with conventional CMOS process. Different numbers of SiNWs which are single, 10 arrays of nanowires and 20 arrays of nanowires were developed. Subsequently, the two metal electrodes which are designated as source (S) and drain (D) were fabricated on top of individual SiNWs using optical lithography process. Optical and electrical characteristic have been proposed to verify the outcome of the fabricated structures. One major part is to observe the SiNWs optically in order to meet the nano-scale variation by using High Power Microscope (HPM) inspection and Field Emission Scanning Electron Microscope (FESEM) imaging. Finally, the samples will be tested electrically using I-V measurement system. The results show that device with single SiNW with $60 \mathrm{~nm}$ in width give the highest resistivity value due to surface to volume ratio.
\end{abstract}

Keyword: Electrical characterization; Electron beam lithography; Silicon nanowires 